\title{
Fractal FSS: \\ A Novel Dual-Band Frequency Selective Surface
}

\author{
Jordi Romeu, Member, IEEE, and Yahya Rahmat-Samii, Fellow, IEEE
}

\begin{abstract}
The multiband properties of self-similar fractals can be advantageously exploited to design multiband frequency selective surfaces (FSS). A Sierpinski dipole FSS has been analyzed and measured and the results show an interesting dual-band behavior. Furthermore a near-field measurement technique is applied to characterize the FSS response to different angles of incidence. Finally, it will be shown that it is possible to tune the FSS response by properly perturbating the geometry of the Sierpinski dipole.
\end{abstract}

Index Terms-Fractals, frequency selective surface.

\section{INTRODUCTION}

$\mathbf{T}$ HE USE of frequency selective surfaces has been successfully proven as a mean to increase the communication capabilities of satellite platforms. In space missions such as Voyager, Galileo, and Cassini, the use of dual-reflector antennas with a subreflector made of an FSS has made it possible to share the main reflector among different frequency bands [1]-[4]. The increasing demands on multifunctionality of antennas for communications require complex FSS with multiband requirements.

Complex multiband FSSs are the result of one, or the combination of several of the following techniques (see Fig. 1): layered or stacked FSS, perturbation of a single-layered FSS [5], or the use of multiresonant elements such as the concentric rings [6]. The Cassini FSS is an example of a complex structure where two single-layer FSS with multiresonant elements are stacked to obtain the desired performance [7]. In practice, the use of multiresonant elements results in a lighter structure and a simplified design.

Fractal shapes have some interesting properties [8]. One of them is the possibility to obtain an arbitrarily long curve confined in a given volume. This property has been shown effective in reducing the spacing between resonant elements in an FSS [9] and in reducing the volume occupied by small antennas [10]. Another interesting property is the self-similarity property. In plain words self-similarity can be described as the replication

Manuscript received August 3, 1999; revised February 16, 2000. This work was done in part while J. Romeu was on sabbatical leave at a NATO Scientific Committee Fellowship. This work was supported by the U.S./Spain Science and Technology Program 1999 under Grant 99217.

J. Romeu is with the Department of Signal Theory and Communications, Telecommunication Engineering School of the Universitat Politecnica de Catalunya, 08034 Barcelona, Spain (e-mail: romeu@tsc.upc.es).

Y. Rahmat-Samii is with the Department of Electrical Engineering, University of California Los Angeles, Los Angeles, CA 90025 USA (e-mail rahmat@ee.ucla.edu).

Publisher Item Identifier S 0018-926X(00)06926-X.

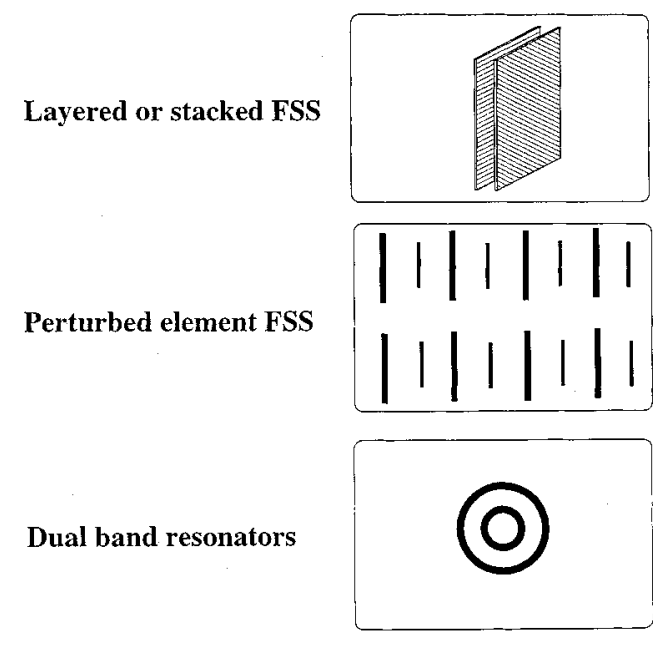

Fig. 1. Possible dual-band FSS configurations.

of the geometry of the structure at a different scale within the same structure. Self-similarity of the structure results in a multiband behavior. The multiband behavior of the fractal Sierpinski dipole has been presented and discussed in [11], [12], [13]. The analysis and results showed that the antenna had a log-periodic behavior. The log-period being related to the self-similarity scale factor of the antenna.

This paper will present the numerical and experimental results obtained from an FSS designed and built by arraying a two-iteration Sierpinski dipole. While some preliminary results were discussed by the authors in [14], this paper will provide an in depth insight to the behavior of the fractal FSS, and the possible ways to modify its response to match it to given specifications. In Section II the main properties of the Sierpinski dipole are reviewed and the design of the FSS is presented. The main limitation to design a truly multiband FSS is the appearance of grating lobes. To avoid grating lobes the spacing between adjacent elements has to be smaller than the free-space wavelength; however, the elements cannot be brought closer than its own length. Although the Sierpinski dipole presents multiple resonances, it will be shown that for a nondielectric backed Sierpinski FSS, only a dual-band behavior can be expected. In Section III the numerical results are presented. To highlight the dual-band behavior on the Sierpinski FSS, its behavior is compared to a bow-tie FSS with analogous dimensions. The plot of the field reflection coefficient in the polar plane shows the dual-resonant characteristic of the Sierpinski 
FSS. The experimental results are shown in Section IV. Two different kinds of measurements were performed. A far-field and a bipolar near-field transmission measurement. The near-field measurement technique is a powerful and simple way to obtain the response of the FSS at a single frequency and for different angles of incidence. The Sierpinski dipole FSS exhibits an interesting dual-band behavior, but in order to be useful it is necessary to tune its response. In Section V two different ways to alter the frequency response of the Sierpinski dipole will be discussed.

\section{SIERPINSKI DIPOLE FREQUENCY SELECTIVE SURFACE}

The Sierpinski dipole is built after the Sierpinski gasket or Sierpinski triangle [15]. The gasket can be constructed by subtracting a central inverted triangle from the original triangle. The process can be successively iterated in the remaining triangles. After infinite iterations the ideal fractal shape is obtained. The resulting object is a self-similar structure [16]. As described in [13] a five iteration Sierpinski dipole presents multiple resonances. The first two frequencies of resonance are given by

$$
2 * h / \lambda_{1}=0.306 \quad 2 * h / \lambda_{2}=1.032
$$

where $2 * h$ is the total height of the dipole and $\lambda_{1}$ and $\lambda_{2}$ are the free-space wavelengths. While the first two resonant frequencies are spaced by a factor of approximately 3.5, the next resonances are spaced by a factor of two-one from the other. In fact, the next resonant frequencies are

$$
2 * h / \lambda_{3}=2.09 \quad 2 * h / \lambda_{4}=4.11 \text {. }
$$

The limiting factor in the high-frequency operation of the FSS is the appearance of the grating lobes. As it happens, in array antennas, grating lobes are responsible for the scattering of energy in undesired directions. For normal incidence, the spacing between adjacent elements should be less than a wavelength in free-space. For large incident angles the spacing should be smaller and the grating lobes are not present for any incident angle when the spacing is smaller than half a wavelength. Unfortunately, the minimum spacing between elements is limited by the own dimensions of the elements. According to the layout of Fig. 2, a triangular lattice is used where the spacing of the elements is chosen such that

$$
d x=d y=2 * d x_{1}=2 * d y_{1} .
$$

The cutoff frequency of the grating lobes depends on the peridiocity of the FSS as well as the direction of the incident field. For a geometry defined as in Fig. 2, and for normal incidence the grating lobes will appear when the following condition is met:

$$
\left(\frac{2 \pi}{\lambda_{p q \text { cutoff }}}\right)^{2}=\left(\frac{2 \pi p}{d x}\right)^{2}+\left(\frac{2 \pi q}{d y_{1}}-\frac{2 \pi p d x_{1}}{d x d y_{1}}\right)^{2}
$$

where $p$ and $q$ are integer indexes and $\lambda_{p q \text { cutoff }}$ is the freespace wavelegth of the cutoff frequency of the grating lobe $p, q$. For the spatial peridiocity of (3) and for normal incidence, the grating lobes will appear when

$$
\frac{2 \pi}{\lambda_{p q \text { cutoff }}}=\frac{2 \pi}{d y} \sqrt{2 p^{2}+(2 q)^{2}-2 p q} .
$$

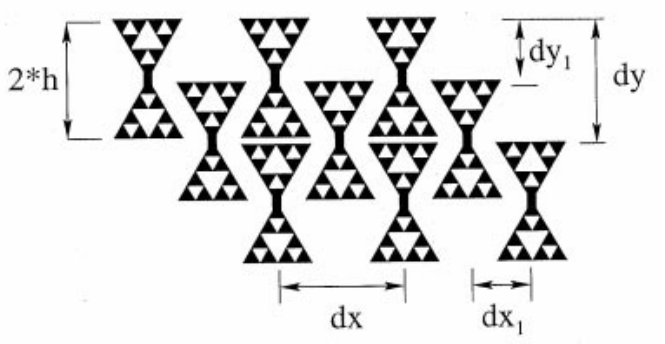

Fig. 2. Geometry of the two iteration Sierpinski dipole and the FSS.

Four degenerated grating lobes appear with indexes $(-1,-1),(-1,0),(1,0),(1,1)$ with a cutoff wavelength given by

$$
\lambda_{\text {cutoff }}=\frac{d y}{\sqrt{2}}
$$

In order to keep the elements from overlapping, $d y$ has to be greater than $2 * h$. When the resonant frequencies of the Sierpinski dipole given by (1) and (2) are considered, it is clear that only the first two resonances of the Sierpinski dipole will occur before the grating lobes in the structure appear. In the design, the value of $d y=1.058 *(2 * h)$ has been chosen. With this value the grating lobes for normal incidence will appear for

$$
(2 * h) / \lambda_{\text {cutoff }}=1.37
$$

that is a frequency above the second resonance of the Sierpinski dipole but below the third resonant frequency. Therefore, for a free-standing Sierpinski dipole FSS only, a dual-band behavior is possible. When a dielectric loading is used, it is possible (as it will be shown in Section IV) to obtain lower resonant frequencies for a given dipole height. Therefore, it can be envisioned a dielectric backed structure with multiband behavior.

\section{ANALYSIS AND NUMERICAL RESUlTS}

The FSS shown in Fig. 2 has been numerically analyzed. The techniques for analyzing frequency selective surfaces are reviewed in [17]. The analysis method is based on the Floquet mode decomposition of the scattered field and the solution by the method of moments technique [18]. The frequency response of the FSS is efficiently computed over a wide frequency range by interpolating the impedance matrix [19]. As it is pointed out in [20] the representation of the reflection coefficient in a polar plane gives a physical insight into the behavior of the FSS. For normal incidence and in the absence of grating lobes a very simple equivalent circuit model can be developed [21], where the FSS is represented by shunt-lumped circuit impedances in a transmission line. For a dipole-like element a series $L-C$ resonant circuit is a good model. When the field reflection coefficient is plotted in the complex plane, the double resonant nature of the FSS is evident. Fig. 3 shows the results obtained after the numerical analysis from 1 to $29 \mathrm{GHz}$ for a Sierpinski dipole FSS when the element height is $2 * h=1.73 \mathrm{~cm}$. The results have been split in two bands, from 1 to $17 \mathrm{GHz}$ is the lower band and from 18 to $29 \mathrm{GHz}$ is the upper band. The Smith chart presenta- 


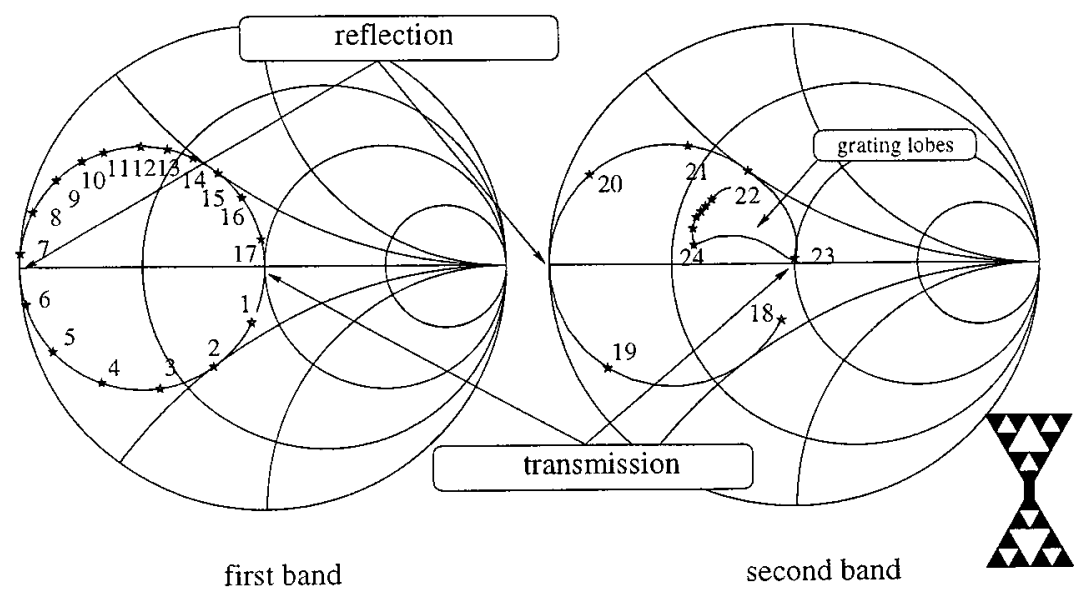

Fig. 3. Field reflection coefficient for normal incidence on the Sierpinski FSS plotted in a Smith Chart. Two resonant bands are clearly formed with resonant frequencies at 6.98 and $19.5 \mathrm{GHz}$. At $23 \mathrm{GHz}$, the grating lobes are present in the structure. The numerically computed data are for an element length of $2 * h=1.73$ $\mathrm{cm}$.

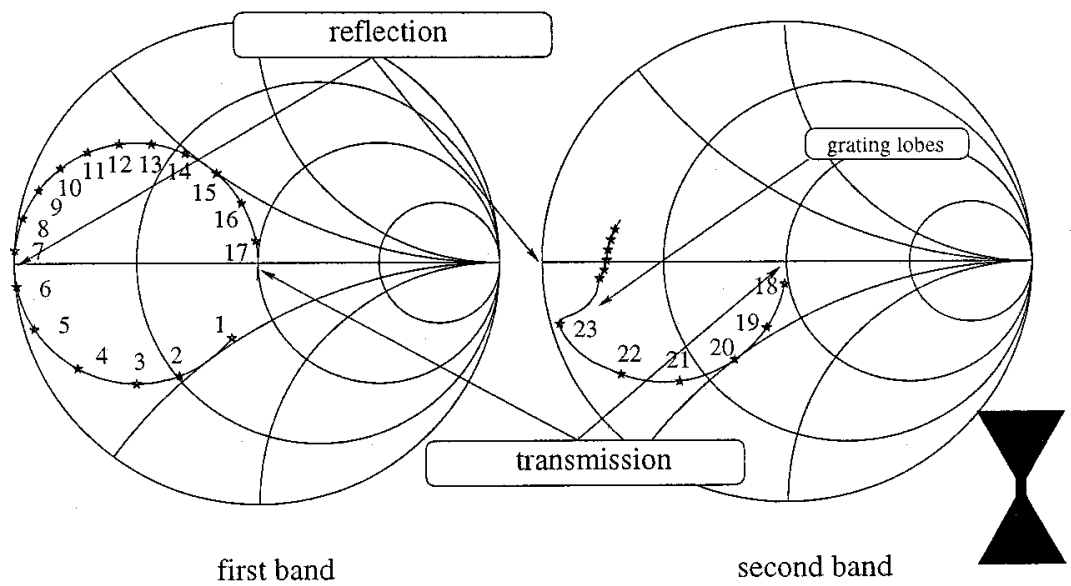

Fig. 4. Field reflection coefficient for normal incidence on the bow-tie FSS. The first band exhibits a similar behavior to the Sierpinski FSS, but the second harmonic resonance is not excited with normal incidence.

tion shows the two resonant frequencies with total reflection at 6.98 and $19.5 \mathrm{GHz}$. These resonant frequencies correspond to

$$
2 * h / \lambda_{1}=0.40 \quad 2 * h / \lambda_{2}=1.13
$$

The values of the resonant frequencies are somewhat higher than predicted in equation (1), probably due to the fact that the values reported in [13] are for a dipole printed on a dielectric substrate. At $23 \mathrm{GHz}$ the grating lobes appear and the simple equivalent circuit model is not valid anymore. The double resonant nature of the Sierpinski FSS is clearly highlighted when it is compared with a bow-tie FSS. The bow-tie FSS has the same dimensions as the Sierpinski FSS, but the Sierpinski dipole has been replaced by a bow-tie dipole of the same height. The results shown in Fig. 4 for the bow-tie FSS clearly denote that the second harmonic resonance of the bow-tie is not excited for normal incidence.

The different behavior of the Sierpinski and the bow-tie FSS is explained by the self-similarity of the Sierpinski gasket. As it was shown in [13], the Sierpinski dipole presents a log-periodic behavior. The number of log-periods is directly related to the number of the iterations in the gasket. The pattern and the input impedance of the Sierpinski dipole has this log-periodic behavior as a result of the self-similar nature of the gasket, which is clearly manifested when the current distribution on the Sierpinski dipole is computed at the different resonant frequencies. On the other hand, the bow-tie presents multiple harmonic resonances, but at each resonance the pattern and the input impedance changes drastically; consequently, when the bow-tie dipole is used in the construction of a FSS a second unit reflection coefficient is not manifested.

The behavior of the Sierpinski dipole FSS can be modeled by a double resonant circuit. Fig. 5 shows the magnitude of the transmission coefficient of the FSS compared to the transmission coefficient over a $50-\Omega$ transmission line of a double resonator. The lumped-element model gives a good prediction of the FSS behavior around the resonances. This model further amplifies the double-resonant nature of the Sierpinski dipole FSS. 


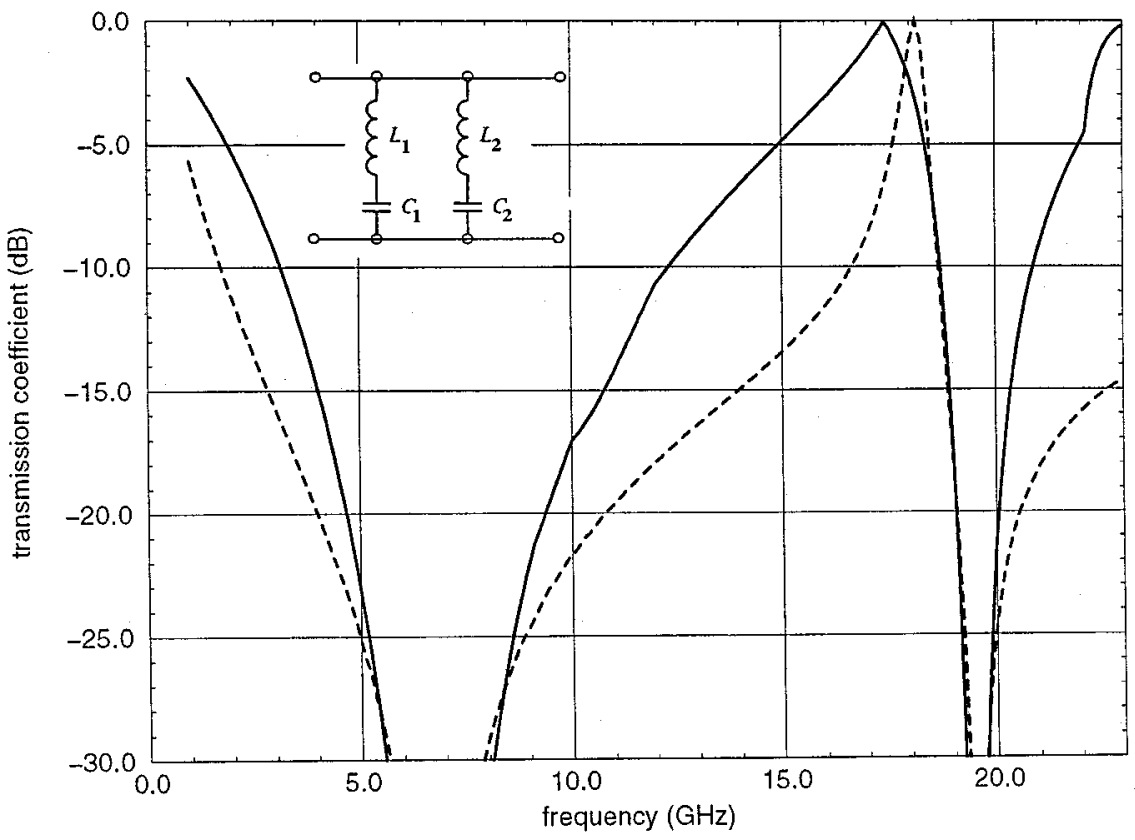

Fig. 5. Comparison of the computed results and the lumped circuit model. The solid line shows the computed data and the dashed line the equivalent circuit model over a 50- $\Omega$ transmission line. The lumped-element values are $C_{1}=7 \mathrm{pF}, L_{1}=74.3 \mathrm{pH}, C_{2}=0.21 \mathrm{pF}$, and $L_{2}=0.317 \mathrm{nH}$.

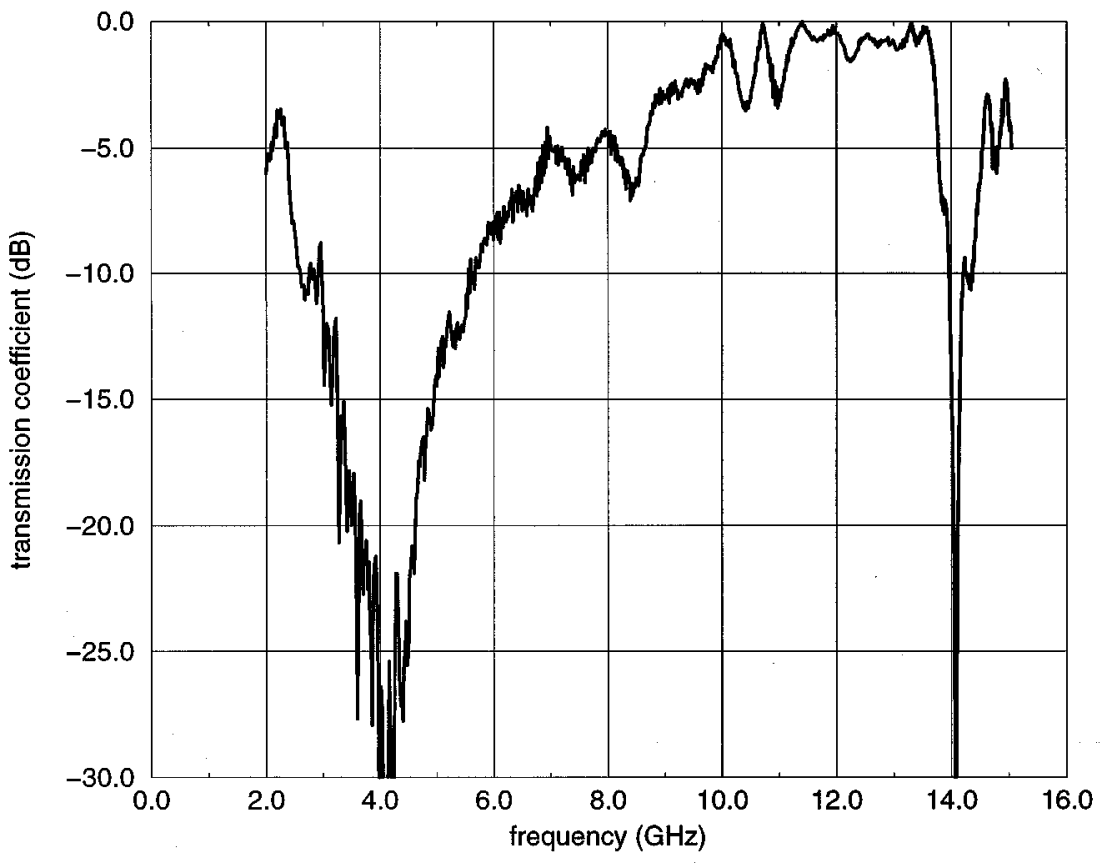

Fig. 6. Transmission coefficient of the Sierpinski dipole FSS with height $2 * h=1.95 \mathrm{~cm}$. The measurements were done from 2 to $15 \mathrm{GHz}$. The resonant frequencies are at 4 and $14 \mathrm{GHz}$. The dielectric backing resulted in lower resonant frequencies in comparison to resonant frequencies of Fig. 5.

\section{MEASUREMENTS}

\section{A. Transmission Measurement}

In order to experimentally verify the behavior of the Sierpinski FSS, a $19 \times 12$ element screen was etched on a 62 mil CuClad substrate $\left(\epsilon_{r}=2.6\right)$. The height of the element was chosen as $2 * h=1.95 \mathrm{~cm}$. The size of the surface was $19 * 25$ $\mathrm{cm}$. The transmission properties of the screen were measured on an antenna range from 2 to $15 \mathrm{GHz}$. In order to perform the measurements, the FSS was placed at distance of $10 \mathrm{~cm}$ in front of a wide-band ridge horn antenna. The measured transmission response was simply calibrated against the transmission response of a dielectric sheet of the same material and thickness. The main difference between the measured and the simulated FSS 


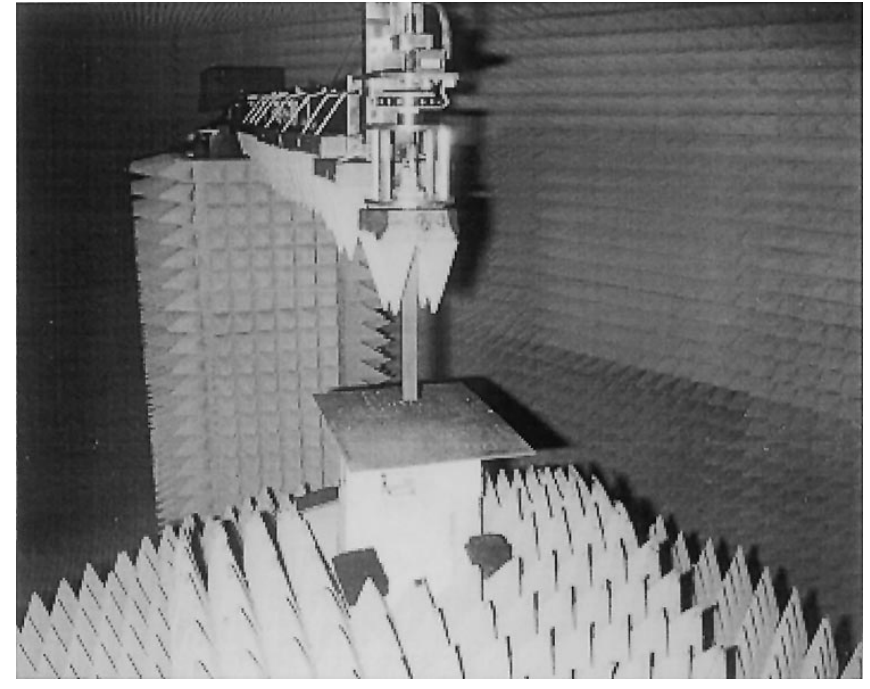

Fig. 7. Measurement setup at the UCLA bipolar near-field range. The fractal FSS is above the horn aperture at a distance of $10 \mathrm{~cm}$. A styrofoam spacer is used to hold the FSS. The near-field probe is at a height of $2.5 \mathrm{~cm}$ from the FSS.

is the presence of the dielectric backing in the former. It is well known that the effect of the dielectric is a reduction of the resonant frequency and the bandwidth [22]. A simple way to model the effect of the dielectric substrate is to increase the value of the capacitors in the lumped element model by a factor $\epsilon_{\mathrm{eff}}$, where $\epsilon_{\text {eff }}$ denotes a relative effective permittivity. As a first approximation it can be assumed that $\epsilon_{\mathrm{eff}}=\left(1+\epsilon_{r}\right) / 2$. By considering this correction and the resonant frequencies expressed in (1), the following resonant frequencies should be expected for the dielectric backed FSS

$$
2 * h / \lambda_{1}=0.29 \quad 2 * h / \lambda_{2}=0.84
$$

The measured results of Fig. 6 show the resonant frequencies at 4 and $14 \mathrm{GHz}$, respectively, which correspond to $2 * h / \lambda_{1}=$ 0.26 and $2 * h / \lambda_{2}=0.93$. These values are within a $10 \%$ error from the ones predicted in (9). At the resonant frequencies the FSS presents transmission nulls deeper than $30 \mathrm{~dB}$.

\section{B. Near-Field Measurements}

It is also interesting to characterize the behavior of the FSS for different angles of incidence. This is almost mandatory in the upper band since the cutoff frequency for the grating lobes diminishes as the angle of incidence moves from broadside. A near-field measurement technique is proposed to obtain the FSS response for different angles of incidence at a given frequency. The measurement scheme consists in performing first a near-field measurement of a rectangular horn antenna and then a second measurement with the FSS placed at a distance of $10 \mathrm{~cm}$ of the horn aperture. With the first measurement, the far field of the horn antenna is obtained. This far field is directly related to the plane wave spectrum (PWS) of the antenna radiated fields. Let $\vec{t}_{\text {horn }}(u, v)$ denote the antenna PWS. The far field can be written as

$$
\vec{E}_{\mathrm{horn}}(\vec{r})=j \frac{e^{-j k r}}{r} k \cos \theta \vec{t}_{\mathrm{horn}}(u, v)
$$
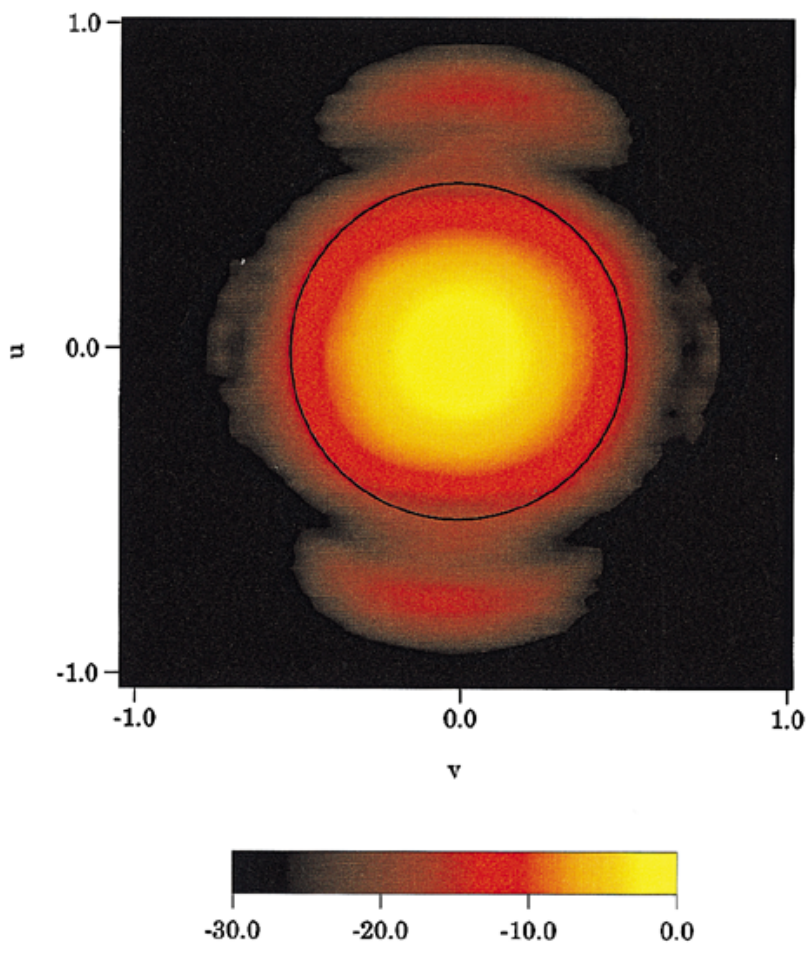

dB

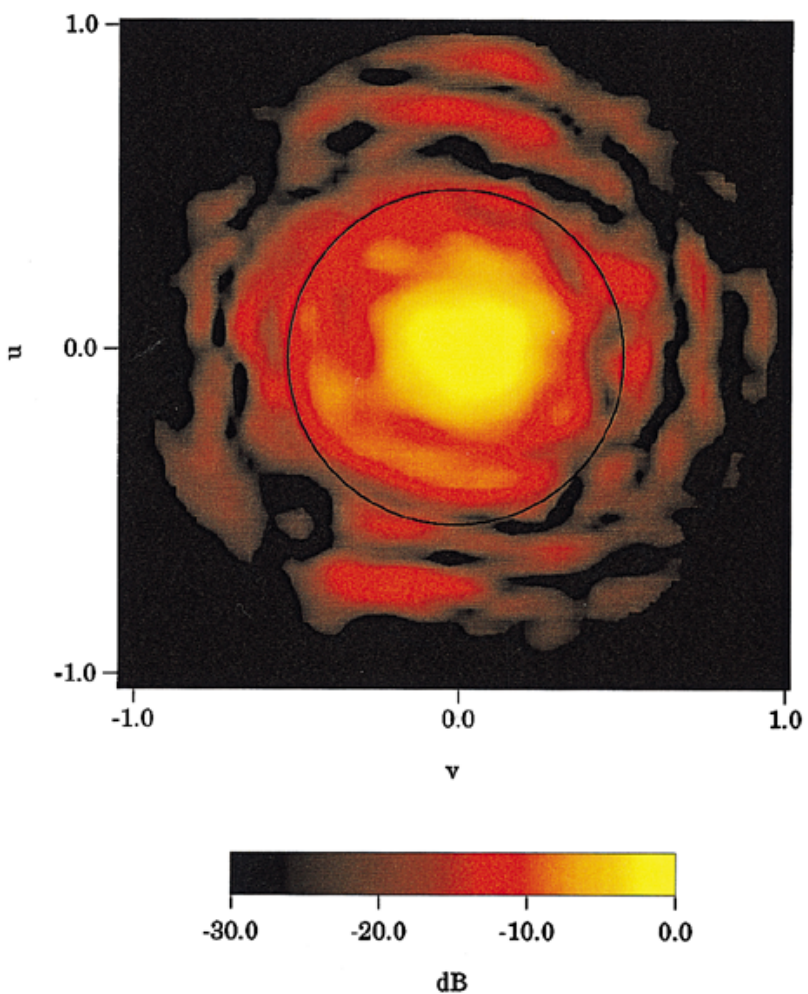

Fig. 8. The top figure shows the far-field pattern of a rectangular horn at 13.27 $\mathrm{GHz}$, and the bottom figure shows the pattern for the same antenna at the same frequency when Sierpinski dipole FSS is placed at $10 \mathrm{~cm}$ from the horn aperture. The circumference shows the valid angle of the far-field pattern constructed from the near field data.

where

$$
u=\sin \theta \cos \phi \quad v=\sin \theta \sin \phi
$$



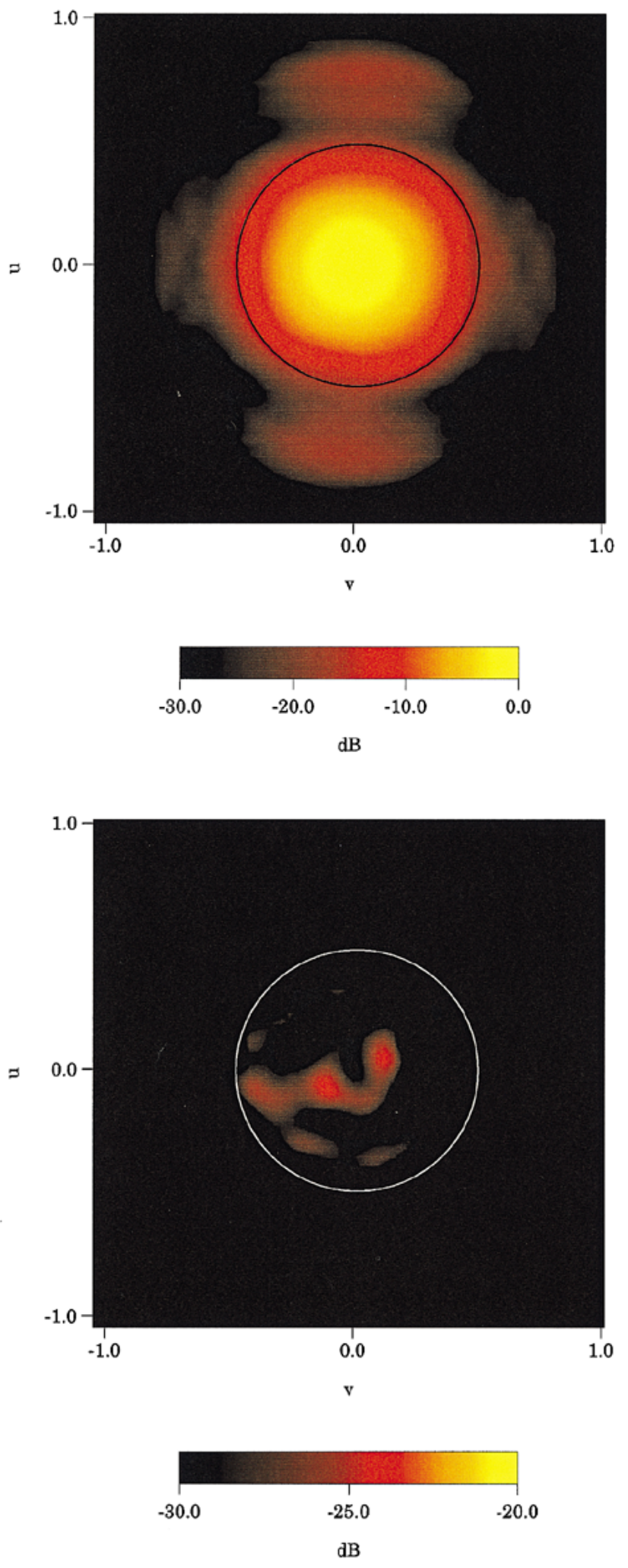

Fig. 9. Comparison of the horn pattern at $14.08 \mathrm{GHz}$ with (top) and without (bottom) FSS. This frequency corresponds to a stop band. Note the change of scale of the bottom figure. In contrast to the results shown in Fig. 8, the FSS practically blocks the horn radiation.

are the cosine directors of the direction of propagation of the different components of the PWS. The response of the FSS as a function of the incident angle can be written as the tensor
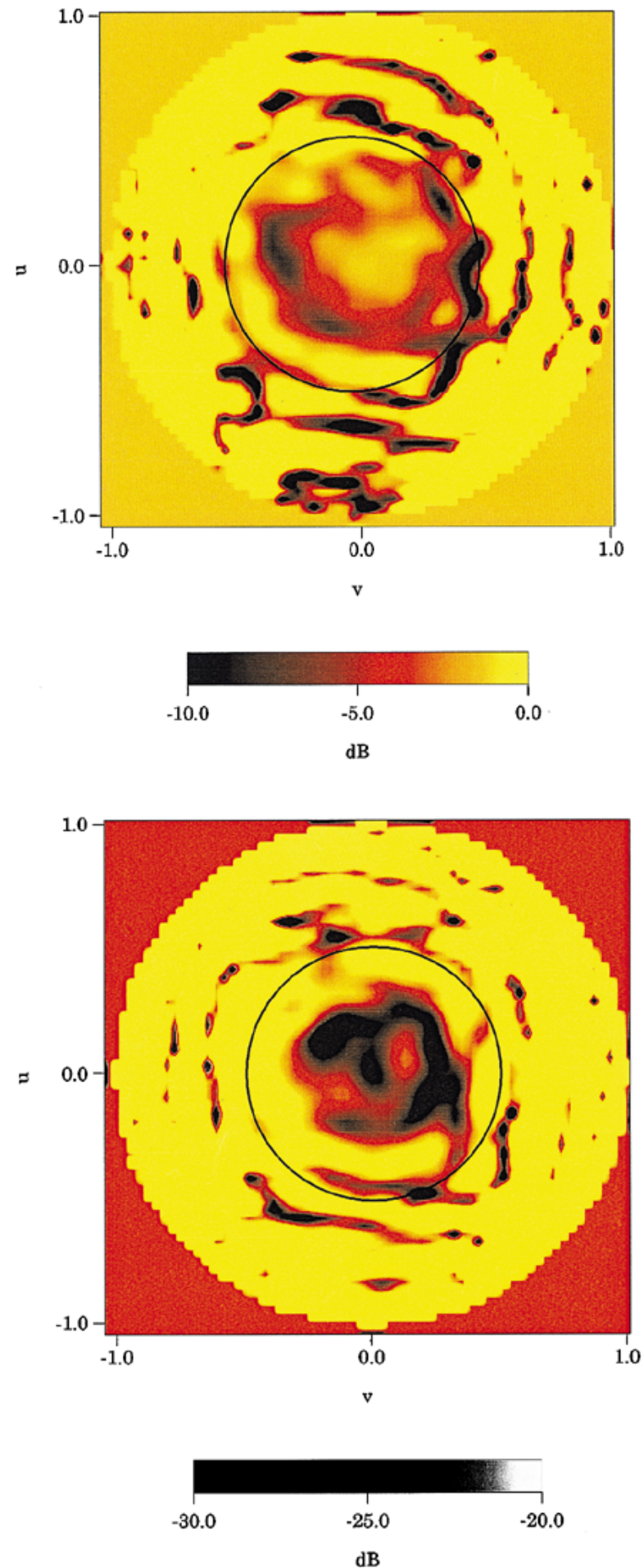

Fig. 10. (a) Response of the FSS at $13.27 \mathrm{GHz}$ and (b) $14.08 \mathrm{GHz}$. At the stop band (b) the FSS response presents an attenuation greater than $25 \mathrm{~dB}$ for incident waves of less than $17^{\circ}$ from broadside. Note the different scales used to show the response in the transmission and stop band.

$\bar{T}(u, v)$ and the far field of the horn with the FSS in front can be written as

$$
\vec{E}_{h o r n+\mathrm{FSS}}(\vec{r})=j \frac{e^{-j k r}}{r} k \cos \theta \vec{t}_{\mathrm{horn}}(u, v) \cdot \bar{T}(u, v)
$$



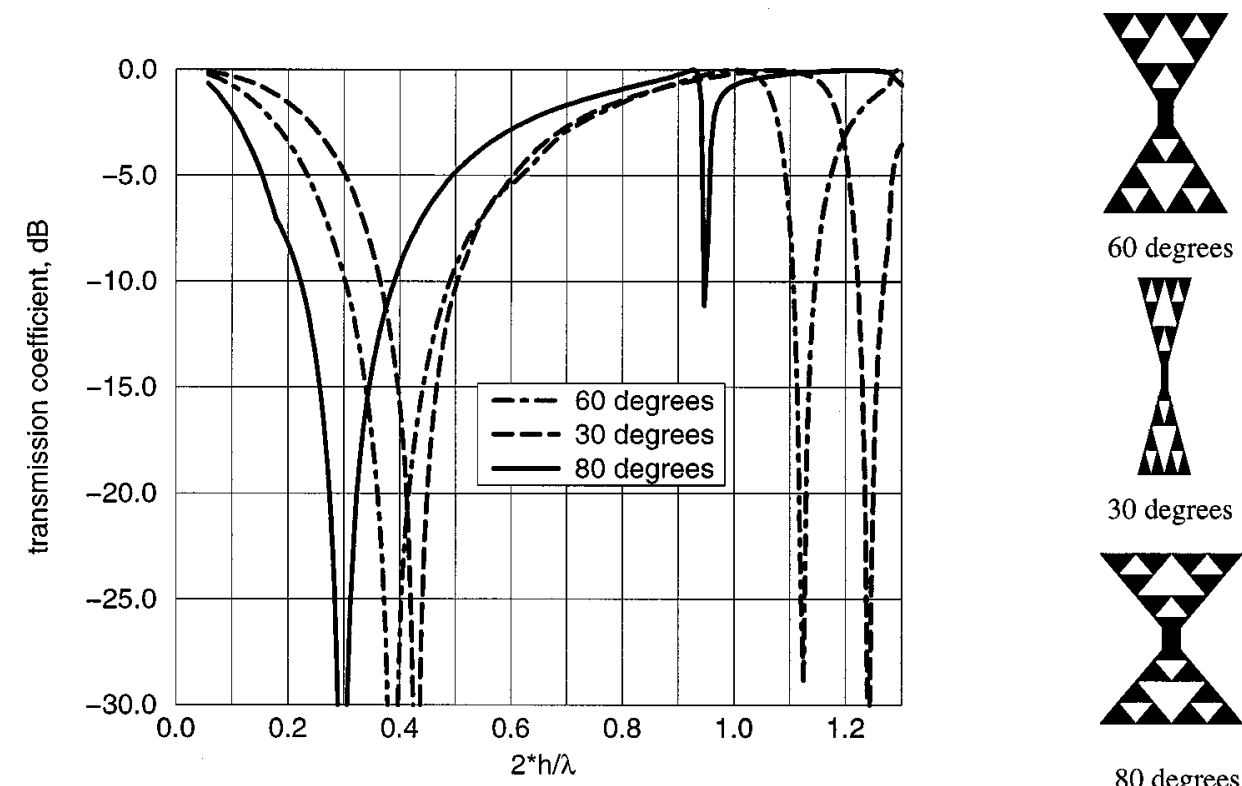

60 degrees

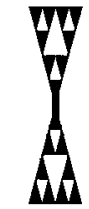

30 degrees

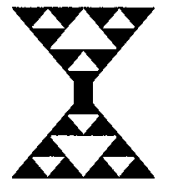

80 degrees

Fig. 11. Comparison of the transmission coefficient for the Sierpinski dipole FSS with different flare angle. The wider the flare angle, the lower the resonant frequencies
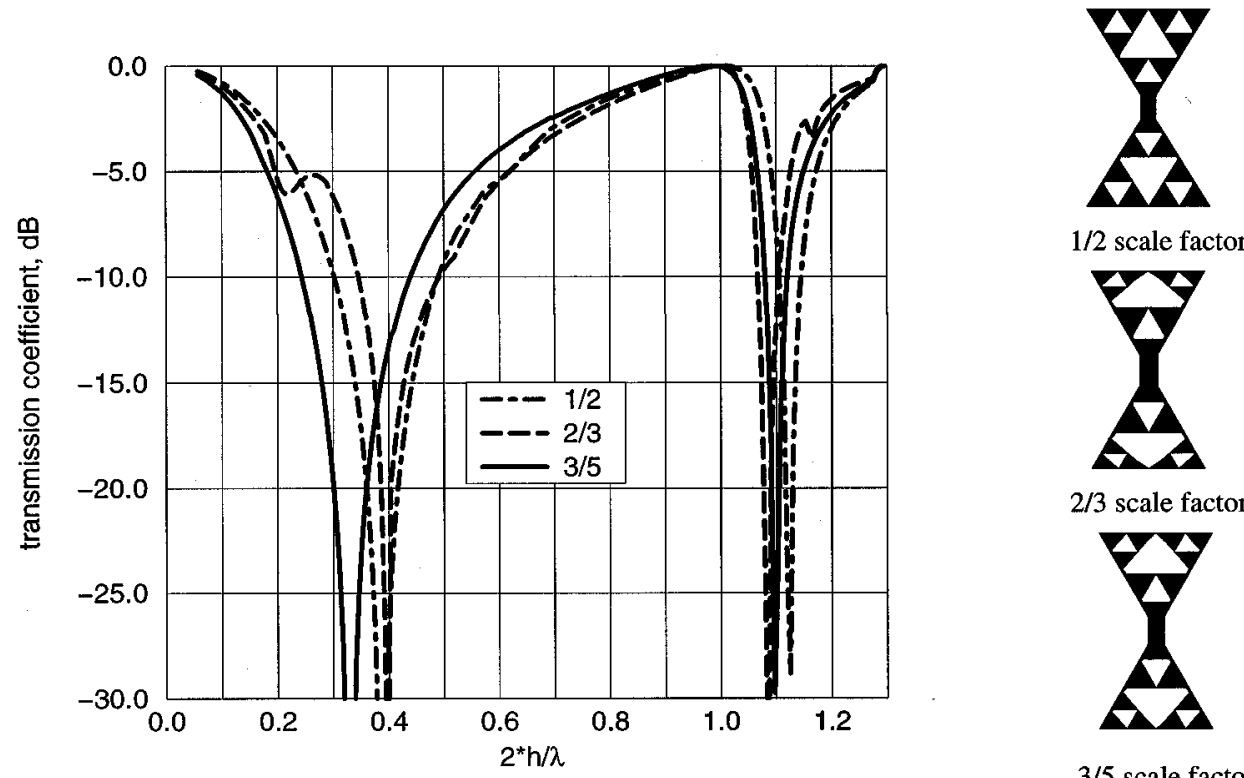

$2 / 3$ scale factor

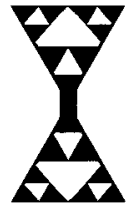

$3 / 5$ scale factor

Fig. 12. Comparison of the transmission coefficient for the Sierpinski dipole FSS with different scale factors. The perturbation of the Sierpinski dipole modifies the ratio between resonant frequencies.

Assuming no significant depolarization by the FSS and very low cross-polar level in the horn radiated fields, the response of the FSS can be obtained as

$$
T^{c c}(u, v)=\frac{E_{\text {horn }+\mathrm{FSS}}^{c}}{E_{\text {horn }}^{c}}
$$

where the index $c$ denotes the copolar component.

The measurements were made at the UCLA bipolar near field range [24]. Fig. 7 shows the measurement setup. Fig. 8 shows the results at a frequency of $13.27 \mathrm{GHz}$. At this frequency the
FSS has a bandpass transmission characteristic. The top of the figure shows the far field of the horn in the $u-v$ plane, and the bottom one the far field of the horn with the FSS. Fig. 9 shows the measurements at $14.08 \mathrm{GHz}$, that is, the second resonant frequency of the FSS. In all figures, the circle indicates the validity region of the far-field data as a result of the near to far-field transformation. In Fig. 10 the response of the FSS computed as indicated in (13) is shown. The results show that for values

$$
u^{2}+v^{2}<0.3^{2}
$$


the transmission loss at $13.27 \mathrm{GHz}$ is less than a fraction of decibels, while the transmission loss at the stop frequency of $14.08 \mathrm{GHz}$ is greater than $25 \mathrm{~dB}$. These values are obtained for an incident angle of less than $17^{\circ}$ from broadside.

\section{TUNING OF THE RESPONSE}

The Sierpinski dipole FSS shows an interesting dual-band behavior, but in order to be useful for practical applications it is necessary to tune its response. By tuning it is meant to change the resonant frequencies and the ratio between them. The results presented so far are for a $60^{\circ}$ Sierpinski dipole. As in the case of a bow-tie antenna [23] it is possible to modify the resonant frequency of the Sierpinski dipole by changing the flare angle [25]. For the Sierpinski dipole it is known that the resonant frequencies are associated with the length of the dipole edge rather than to its height. In consequence, for a given height as the flare angle of the dipoles becomes wider the frequencies of resonance become lower. Fig. 11 shows a comparison of the transmission coefficient for Sierpinski dipole FSS with dipoles with flare angles of $30^{\circ}, 60^{\circ}$, and $80^{\circ}$. The geometry of the FSS is the same as shown in Fig. 2 and only the flare angle of the Sierpinski dipole has been modified. As expected, the results show that as the flare angle widens the resonant frequency diminishes. It is also interesting to note that the factor between the first and the second resonance remains almost unaltered in the three cases.

As shown in [26], it is possible to modify the ratio between resonance frequencies in the Sierpinski dipole by properly perturbating its shape. The Sierpinski gasket as described in Section II has a scale ratio between one triangle and the triangles obtained in the next iteration of one half. This scale factor can be altered to modify the ratio between resonant frequencies. Fig. 12 shows the transmission coefficient for a Sierpinski dipole FSS with different scale factors. Although it is not possible to relate the scale factor to the ratio of the resonant frequencies, the results show that to a certain extent it is possible to tune the response of the FSS.

\section{CONCLUSION}

It has been shown that the self-similarity of the Sierpinski dipole translates into a dual-band behavior of a FSS made by arraying a two iteration Sierpinski dipole. The dual-band behavior is ultimately the result of the self-similarity properties of the Sierpinski gasket that allows to embed in its geometry resonators at different frequencies.

The experimental results are in good agreement with the numerical analysis. A near-field measurement technique has been successfully applied to characterize the response of the FSS at different angles of incidence. The geometry of the Sierpinski dipole offers sufficient degrees of freedom as to make possible to modify its shape in order to tune its response.

\section{REFERENCES}

[1] G. H. Schennum, "Frequency-selective surfaces for multiple frequency antennas," Microwave J., vol. 16, pp. 55-57, May 1973.
[2] Y. Rahmat-Samii and M. Gatti, "Far-field patterns of space-borne antennas from near-field measurements," IEEE Trans. Antennas Propagat., vol. AP-33, pp. 638-648, June 1985.

[3] Y. Rahmat-Samii and A. N. Tulintseff, "Diffraction analysis of frequency selective reflector antennas," IEEE Trans. Antennas Propagat., vol. 41, pp. 476-487, Apr. 1993.

[4] T. K. Wu, "Cassini frequency selective surface development," J. Electromagn. Waves Applicat., vol. 8, no. 12, pp. 1547-1561, Dec. 1994.

[5] R. A. Hill and B. A. Munk, "The effect of perturbating a frequency selective surface and its relation to the design of a dual-band surface," IEEE Trans. Antennas Propagat., vol. 44, pp. 368-374, Mar. 1996.

[6] E. A. Parker and J. C. Vardaxoglou, "Plane-wave illumination of concentric-ring frequency-selective surfaces," Proc. Inst. Elect. Eng.-Microwaves, Opt., Antennas), pt. H, vol. 132, no. 3, pp. 176-180, June 1985.

[7] T.-K. Wu and S.-W. Lee, "Multiband frequency surface with multiring patch elements," IEEE Trans. Antennas Propagat., vol. 42, no. 11, pp. 1484-1490, Nov. 1994.

[8] D. H. Werner and R. Mittra, Eds., Frontiers in Electromagnetics. Piscataway, NJ: IEEE Press, 1999, ch. 1-3.

[9] E. A. Parker and A. N. A. El Sheikh, "Convoluted array elements and reduced size unit cells for frequency-selective surfaces," Proc. Inst. Elect. Eng.-Microwaves, Opt., Antennas), pt. H, vol. 138, no. 1, pp. 19-22, Feb. 1991.

[10] C. Puente, J. Romeu, R. Pous, J. Ramis, and A. Hijazo, "Small but long Koch fractal monopole," Inst. Elect. Eng. Electron. Lett., vol. 34, no. 1, pp. 9-10, Jan. 1998.

[11] C. Puente, "Fractal antennas," Ph.D. dissertation, Dept. Signal Theory Commun., Univ. Politecnica de Catalunya, Spain, June 1997.

[12] C. Puente, J. Romeu, R. Pous, X. Garcia, and F. Benitez, "Fractal multiband antenna based on the Sierpinski gasket," Electron. Lett., vol. 32, no. 1, pp. 1-2, Jan. 1996.

[13] C. Puente, J. Romeu, R. Pous, and A. Cardama, "On the behavior of the Sierpinski multiband antenna," IEEE Trans. Antennas Propagat., vol. 46, no. 4, pp. 517-524, Apr. 1998.

[14] J. Romeu and Y. Rahmat-Samii, "Dual band FSS with fractal elements," Electron. Lett., vol. 35, no. 9, pp. 702-703, Apr. 1999.

[15] H. O. Peitgen, H. Jurgens, and D. Saupe, Chaos and Fractals, New Frontiers in Science. New York: Springer-Verlag, 1992.

[16] J. E. Hutchinson, "Fractals and self-similarity," Indiana Univ. Math. J., vol. 30, pp. 713-747, 1981.

[17] R. Mittra, C. H. Chan, and T. Cwik, "Techniques for analysing frequency selective surfaces-A review," Proc. IEEE, vol. 76, pp. 1593-1615, Dec. 1988.

[18] J. P. Montgomery, "Scattering by an infinite periodic array of thin conductors on a dielectric sheet," IEEE Trans. Antennas Propagat., vol. AP-23, pp. 70-75, Jan. 1975.

[19] A. S. Barlevy and Y. Rahmat-Samii, "An efficient method for wide band characterization of periodic structures using modified $\mathrm{Z}$ matrix interpolation," in 1997 IEEE Antennas Propagat. Soc. Int. Symp. Dig., Montreal, Quebec, Canada, July 1997, pp. 56-59.

[20] - "Fundamental constrain on the electrical characteristics of frequency selective surfaces," Electromagn., vol. 17, no. 1, pp. 41-68, Jan./Feb. 1997.

[21] C. K. Lee and R. J. Langley, "Equivalent circuit models for frequency selective surfaces at oblique angles of incidence," Proc. Inst. Elect. Eng.-Microwaves, Opt., Antennas), pt. H, vol. 132, pp. 395-399, Oct. 1985.

[22] P. Callaghan, E. A. Parker, and R. J. Langley, "Influence of supporting dielectric layers on the transmission properties of frequency selective surfaces," Proc. Inst. Elect. Eng.-Microwaves, Opt., Antennas), vol. 138, no. 5, pp. 448-454, Oct. 1991.

[23] G. H. Brown and O. M. Woodward, "Experimentally determined radiation characteristics of conical and triangular antennas," RCA Rev., pp. 425-452, Dec. 1952.

[24] Y. Rahmat-Samii, L. I. Williams, and R. G. Yaccarino, "The UCLA bi-polar planar near-field antenna measurement and diagnostics range," Antennas Propagat. Mag., vol. 37, no. 6, pp. 16-35, Dec. 1995.

[25] C. Puente, M. Navarro, J. Romeu, and R. Pous, "Variations on the fractal Sierpinski antenna flare angle," in 1998 IEEE Antennas Propagat. Soc. Int. Symp. Dig., Atlanta, GA, June 1998, pp. 2340-2341.

[26] C. Puente, J. Romeu, R. Bartolome, and R. Pous, "Perturbation of the Sierpinski antenna to allocate operating bands," Inst. Elect. Eng. Electron. Lett., vol. 32, no. 24, pp. 2186-2187, Nov. 1996. 


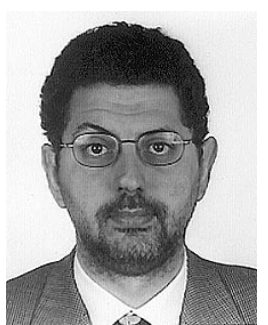

Jordi Romeu (S'88-M'93) was born in Barcelona, Spain, in 1962. He received the Ing. and Dr. Ing. degrees in telecommunication engineering, both from the Polytechnic University of Catalunya (UPC), Barcelona, Spain, in 1986 and 1991, respectively.

In 1985, he joined the Electronagnetic and Photonics Engineering Group, Signal Theory and Communications Department, UPC. Currently, he is Associate Professor there, where he is engaged in research in antenna near-field measurements, antenna diagnostics, and antenna design. He was Visiting Scholar at the Antenna Laboratory, University of California, Los Angeles, in 1999, on a NATO Scientific Program Scholarship. He holds several patents and has published papers in the fields of antenna near-field measurements and diagnostics and in antenna design.

Dr. Romeu was grand winner of the European IT Prize, awarded by the European Commission, for his contributions in the development of fractal antennas in 1998

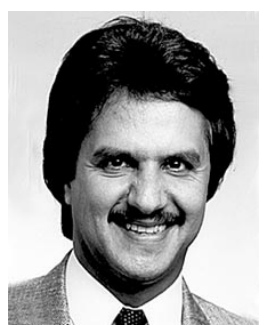

Yahya Rahmat-Samii (S'73-M'75-SM'79-F'85) received the M.S. and Ph.D. degrees in electrical engineering from the University of Illinois, Urbana-Champaign.

Currently, he is a Professor of electrical engineering at the University of California, Los Angeles (UCLA). He was a Senior Research Scientist at NASA's Jet Propulsion Laboratory/California Institute of Technology before joining UCLA. He was a Guest Professor at the Technical University of Denmark (TUD) in the summer of 1986 . He has also been a Consultant to many aerospace companies. He has been Editor and Guest Editor of many technical journals and book publication entities.He has authored and coauthored more than 450 technical journal articles and conference papers and has written 14 book chapters. He is the coauthor of the books entitles, Electromagnetic Optimization by Genetic Algorithms (New York: Wiley), and Impedance Boundary Conditions in Electromagnetics (Wahington, DC: Taylor Francis), published in 1999 and 1995, respectively. He is also the holder of several patents. He has pioneering research contributions in diverse areas of electromagnetics, antennas, measurement and diagnostics techniques, numerical and asymptotic methods, satellite and personal communications and human/antenna interactions, etc.

Dr. Rahmat-Samii was the 1995 President and 1994 Vice-President of IEEE Antennas and Propagation Society. He was appointed an IEEE Antennas and Propagation Society Distinguished Lecturer and presented lectures internationally. He was elected as a Fellow of IAE in 1986. He was a member of the Strategic Planning and Review Committee (SPARC) of IEEE. He has been the Guest and Plenary Session Speaker at many national and international symposia. He was one of the directors and Vice President of the Antennas Measurement Techniques Association (AMTA) for three years. He has also served as Chairman and Co-Chairman of several national and international symposia. Dr. Rahmat-Samii was also a member of UCLA's Graduate council for a period of three years. For his contributions, he has received numerous NASA and JPL Certificates of Recognition. In 1984, he was the recipient of the prestigious Henry Booker Award of URSI. In 1992 and 1995, he was the recipient of the Best Application Paper Award (Wheeler Award) for papers published in the 1991 and 1993 IEEE TRANSACTIONS ON ANTENNAS AND PROPAGATION. He is a member of Commissions A, B, and J of USNC/URLI, AMTA, Sigma Xi, Eta Kappa Nu and Electromagnetics Academy. He is listed in Who's Who in America, Who's Who in Frontiers of Science, and Technology and Who's Who in Engineering. In 1999, he was the recipient of the University of Illinois ECE Distinguished Alumni Award. In 2000 he was selected as the recipient of IEEE Third Millennium Medal. 